\title{
Agrichemicals and antibiotics in combination increase antibiotic resistance evolution
}

\author{
Brigitta Kurenbach $^{1}{ }^{\text {, Amy M Hill }}{ }^{1}$, William Godsoe ${ }^{2}$, Sophie van Hamelsveld ${ }^{1}$, Jack A Heinemann ${ }^{\text {Corresp. } 1}$ \\ ${ }^{1}$ School of Biological Sciences and Centre for Integrated Research in Biosafety and Centre for Integrative Ecology, University of Canterbury, Christchurch, \\ New Zealand \\ 2 Bio-Protection Centre, Lincoln University, Lincoln, New Zealand \\ Corresponding Author: Jack A Heinemann \\ Email address: jack.heinemann@canterbury.ac.nz
}

Antibiotic resistance in our pathogens is medicine's climate change: caused by human activity, and resulting in more extreme outcomes. Resistance emerges in microbial populations when antibiotics act on phenotypic variance within the population. This can arise from either genotypic diversity (resulting from a mutation or horizontal gene transfer), or from differences in gene expression due to environmental variation, referred to as adaptive resistance. Adaptive changes can increase fitness allowing bacteria to survive at higher concentrations of antibiotics. They can also decrease fitness, potentially leading to selection for antibiotic resistance at lower concentrations. There are opportunities for other environmental stressors to promote antibiotic resistance in ways that are hard to predict using conventional assays. Exploiting our previous observation that commonly used herbicides can increase or decrease the minimum inhibitory concentration (MIC) of different antibiotics, we provide the first comprehensive test of the hypothesis that the rate of antibiotic resistance evolution under specified conditions can increase, regardless of whether a herbicide increases or decreases the antibiotic MIC. Short term evolution experiments were used for various herbicide and antibiotic combinations. We found conditions where acquired resistance arises more frequently regardless of whether the exogenous non-antibiotic agent increased or decreased antibiotic effectiveness. This is attributed to the effect of the herbicide on either MIC or the minimum selective concentration (MSC) of a paired antibiotic. The MSC is the lowest concentration of antibiotic at which the fitness of individuals varies because of the antibiotic, and is lower than MIC. Our results suggest that additional environmental factors influencing competition between bacteria could enhance the ability of antibiotics to select antibiotic resistance. Our work demonstrates that bacteria may acquire antibiotic resistance in the environment at rates substantially faster than predicted from laboratory conditions. 


\section{Classification: Biological sciences / evolution}

2

3 Agrichemicals and antibiotics in combination increase antibiotic resistance evolution

5 Brigitta Kurenbach ${ }^{1 \dagger}$, Amy M. Hill ${ }^{1 \dagger}$,William Godsoe ${ }^{2}$, Sophie van Hamelsveld ${ }^{1}$, and Jack A.

6 Heinemann $^{1 *}$

7

$8{ }^{1}$ School of Biological Sciences, University of Canterbury, Christchurch, NZ and Centre for 9 Integrated Research in Biosafety and Centre for Integrative Ecology, University of Canterbury,

10

11

12 13

14

\section{7}

${ }^{2}$ Bio-Protection Centre, Lincoln University, Lincoln, NZ

*corresponding author: Jack A. Heinemann, School of Biological Sciences, University of Canterbury, Christchurch, NZ

Tel.: (+64) 33642500

Email: jack.heinemann@canterbury.ac.nz

$\uparrow \mathrm{BK}$ and $\mathrm{AMH}$ contributed equally to this work.

\section{Short title: Herbicide evolved antibiotic resistance}

\section{Abstract}

Antibiotic resistance in our pathogens is medicine's climate change: caused by human activity, and resulting in more extreme outcomes. Resistance emerges in microbial populations when antibiotics act on phenotypic variance within the population. This can arise from either genotypic diversity (resulting from a mutation or horizontal gene transfer), or from differences in gene expression due to environmental variation, referred to as adaptive resistance. Adaptive changes can increase fitness allowing bacteria to survive at higher concentrations of antibiotics. They can also decrease fitness, potentially leading to selection for antibiotic resistance at lower 
28 concentrations. There are opportunities for other environmental stressors to promote antibiotic

29 30 resistance in ways that are hard to predict using conventional assays. Exploiting our previous observation that commonly used herbicides can increase or decrease the minimum inhibitory concentration (MIC) of different antibiotics, we provide the first comprehensive test of the hypothesis that the rate of antibiotic resistance evolution under specified conditions can increase, regardless of whether a herbicide increases or decreases the antibiotic MIC. Short term evolution experiments were used for various herbicide and antibiotic combinations. We found conditions where acquired resistance arises more frequently regardless of whether the exogenous nonantibiotic agent increased or decreased antibiotic effectiveness. This is attributed to the effect of the herbicide on either MIC or the minimum selective concentration (MSC) of a paired antibiotic. The MSC is the lowest concentration of antibiotic at which the fitness of individuals varies because of the antibiotic, and is lower than MIC. Our results suggest that additional environmental factors influencing competition between bacteria could enhance the ability of antibiotics to select antibiotic resistance. Our work demonstrates that bacteria may acquire antibiotic resistance in the environment at rates substantially faster than predicted from laboratory conditions.

\section{Significance}

Neither reducing the use of antibiotics nor discovery of new ones may be sufficient strategies to avoid the post-antibiotic era. This is because bacteria may be exposed to other non-antibiotic chemicals that predispose them to evolve resistance to antibiotics more quickly. Herbicides are examples of some of the most common non-antibiotic chemicals in frequent global use. We previously showed that in some combinations the herbicides we tested made bacteria phenotypically resistant to higher concentrations of antibiotics, while in other combinations bacteria became susceptible at lower antibiotic concentrations. Here we demonstrate that in both cases the herbicides worked with antibiotics to accelerate genotypic resistance evolution. Unfortunately, antibiotic resistance may increase even if total antibiotic use is reduced, and new ones are invented, unless other environmental exposures are also controlled. 
58 Abbreviations MIC, minimum inhibitory concentration; MSC, minimum selective 59 concentration; NOEL, no observable effect level 
60

61

62

63

64

65

66

67

68

69

70

71

72

73

74

75

76

77

78

79

80

81

82

83

84

85

86

87

88

89

\section{Introduction}

As fundamental tools for infection control, antibiotics underpin diverse human systems ranging from hospital care to concentrated animal feeding operations through to crop and pollinator disease management. The loss of this tool due to antibiotic resistance will result in higher mortality and morbidity, but also deny access to many routine medical procedures for risk of subsequently untreatable infections (Teillant et al. 2015; Thomas et al. 2014). Antibiotic resistances also threaten agricultural productivity (Stockwell \& Duffy 2013; Van Boeckel et al. 2015). Despite over a half century of warning, neither science nor public and private innovation strategies have managed to avert the threat of a post-antibiotic era.

One stewardship strategy is reduction of use which might help increase longevity (CDC 2013; Collingnon et al. 2016). If bacteria almost never encounter antibiotics at concentrations high enough to harm them, there would be little opportunity for resistant variants to emerge and establish. Based on this, it has been suggested that judicious and low use of antibiotics that keeps most antibiotic exposures to less than the minimum inhibitory concentration (MIC) should preserve antibiotic susceptibility in bacteria (Andersson \& Hughes 2014). In practice, the lowest concentration of antibiotic leading to the evolution of resistance in a given environment, the so called minimum selective concentration (MSC), can be much lower than the MIC (Fig. 1) (Andersson \& Hughes 2014; Baquero et al. 1998b; Hermsen et al. 2012). Keeping the use of antibiotics to below MSC concentrations is more challenging still.

Variation in antibiotic responses can be caused by either genetic or physiological differences between individual bacteria. The toxic effect of an antibiotic may occur at different concentrations for different individuals because some have acquired genes or alleles through mutation or horizontal gene transfer (i.e. change in genotype). Also, organisms can have innate differences between them, e.g. due to differences in permeability.

Innate resistance can also be dependent upon genes expressed or repressed conditionally, resulting in increased efflux or decreased influx of antibiotics and overall lower intracellular antibiotic concentrations (Fernandez \& Hancock 2012). Such genes or expression induction thresholds may differ between species, and individuals within a species may phenotypically differ depending on whether or not they expressed those genes before being inhibited by the antibiotic. This resistance through changes in gene expression is also known as an adaptive 
90 (change in phenotype) response (Fernandez et al. 2011) which acclimates bacteria to the

91 environment. It can be triggered by antibiotics and other chemical toxins or environmental cues

92 (Blair et al. 2015; Palmer \& Kishony 2013; Sanchez-Romero \& Casadesus 2014).

93 We have previously shown that concurrent exposure of Escherichia coli or Salmonella enterica

94 sv. Typhimurium to various commercial herbicides and antibiotics from different classes can

95 lead to adaptive increases (Fig. 1A) or decreases (Fig. 1B) in their susceptibility to the

96 antibiotics. In some cases, herbicide exposures changed the survival at below-MIC antibiotic

97 concentrations of antibiotic, while MIC itself was not affected (Fig. 1C). Adaptive responses

98 were specific for the combination of bacteria, antibiotic and herbicide used. Effects were shown

99 for commercial formulations (Kurenbach et al. 2015), for label listed active ingredients, and for

100 common adjuvants (Kurenbach et al. 2017).

101 Populations of adaptively resistant bacteria can in time produce variants with acquired resistance

102 to even higher concentrations of antibiotic (Cohen et al. 1989; Gustafson et al. 1999; Shen et al.

103 2011). This raises the possibility that environmental stimuli that cause phenotypic antibiotic

104 resistance variation between individuals could be hotspots for evolution of acquired antibiotic

105 resistance.

106 Here we exploit our previous observations to test the novel hypothesis that both adaptive

107 increases and decreases in antibiotic susceptibility caused by exposure to herbicide formulations

108 can lead to an increase in the rate of acquired resistance evolution in populations of E. coli and $S$.

109 typhimurium. For this purpose we conducted short term evolution experiments using either the

110 natural variability within a monoculture of E. coli or S. typhimurium, or isogenic strains only

111 differing in their level of antibiotic resistance, which represents natural variability.

\section{Materials and Methods}

\section{Media}

114 Strains and plasmids are listed in Table 1. Bacteria were grown in standard rich growth medium,

115 LB (Lennox) (Invitrogen) at $37^{\circ} \mathrm{C}$ and supplemented with ampicillin (Amp, AppliChem),

116 chloramphenicol (Cam, Sigma) ciprofloxacin (Cip, Pentex), streptomycin (Str, Sigma),

117 tetracycline (Tet, Sigma), or nalidixic acid (Nal, Sigma) as appropriate. Liquid cultures were

118 grown with aeration $(180 \mathrm{rpm})$, and plates were incubated in plastic bags to avoid drying out. 
119 Commercial herbicide formulations used were $\mathrm{Kamba}^{500}$ (Nufarm, NZ) containing $500 \mathrm{~g} / \mathrm{L}$ 120 dimethyl salt of dicamba, and Roundup Weedkiller (Monsanto, Australia) containing $360 \mathrm{~g} / \mathrm{L}$

121 isopropylamine salt of glyphosate. Herbicide concentrations are reported in parts per million acid

122 equivalent ( $\mathrm{ppm}$ ae) of the active ingredient to allow for comparison with other formulations.

123 Antibiotic and herbicide concentrations used are specified in the main text or in figure legends.

124 Plasmid constructs

125 pBR322 (Table 1) was used as the base to create a pair of plasmids that only differed in 126 antibiotic resistance determinants. Plasmid pAH14 was created by deleting a section of the gene

127 for Tet ${ }^{\mathrm{R}}$ by removing the HindIII and Bam HI fragment of pBR322 and inserting cat from

$128 \mathrm{pACYC} 184$ at the PstI site within bla. The resulting plasmid conferred resistance to

129 chloramphenicol, but not to ampicillin or tetracycline $\left(\mathrm{Cam}^{\mathrm{R}}, \mathrm{Amp}^{\mathrm{S}}\right.$, $\left.\mathrm{Tet}^{\mathrm{S}}\right)$. RSF1010 (Table 1)

130 was the base for pAH11, which was created by insertion of $c$ at from pACYC184 into the EcoRI

131 and NotI sites of RSF1010, resulting in a plasmid conferring chloramphenicol but not

132 streptomycin resistance $\left(\mathrm{Cam}^{\mathrm{R}}, \mathrm{Str}^{\mathrm{S}}\right)$.

\section{Culturing conditions}

134 For the experiments described in the section "Evolution of acquired antibiotic resistance in 135 cultures with herbicide-induced increases in MIC" E. coli or S. typhimurium were grown for 24 $136 \mathrm{hrs}$ in $10 \mathrm{~mL}$ liquid LB medium containing Cip, herbicide, both, or neither. Initial densities were 137 ca. $10^{5} \mathrm{cfu} / \mathrm{mL}$ for the former treatment and ca. $10^{6} \mathrm{cfu} / \mathrm{mL}$ (E. coli) and $10^{5} \mathrm{cfu} / \mathrm{mL}(S$. 138 typhimurium) for the latter three. The experiment was abandoned at this point if cultures 139 containing Cip but not herbicide were visibly turbid. This was because in this experiment the 140 chosen Cip concentration was above MIC in cultures not exposed to herbicide, and growth was 141 therefore interpreted as due to a resistant mutant in the starting culture. Herbicide and antibiotic 142 concentrations used are detailed in Table 2 and Table 3. Cultures were then diluted as above in 143 the same conditions and incubated again for $24 \mathrm{hrs}$. A 10-fold dilution in LB without herbicides 144 or antibiotics followed to ensure observed effects were not due to herbicide induced adaptive 145 responses. Titers (cfu/mL) were determined at the end of each incubation step on both LB and on 146 Cip at an initially non-permissive concentration (ca. 2x MIC) (Dan et al. 2016). The frequency of 147 Cip resistant mutants and the number of generations was calculated. 
148 Experiments described in the section "Herbicide-induced changes in MSC can occur without a 149 change in MIC" were conducted similarly. However, in this case the culture supplemented with 150 only Cip was able to grow because the chosen Cip concentration was below MIC.

\section{Determination of resistance levels}

152 Individual colonies of strains grown on solid LB medium were used to inoculate $100 \mu \mathrm{L}$ of liquid 153 LB in a 96 well plate. The plate was incubated with aeration to saturation before ca. $4 \mu \mathrm{L}$ 154 samples were stenciled onto LB plates containing the appropriate antibiotics. Plates were 155 incubated at $37^{\circ} \mathrm{C}$ for $18 \mathrm{hrs}$. Strains were scored positively for growth if growth was tangible.

\section{Mixed culture experiments}

157 Isogenic strains of $E$. coli differing only in the MIC phenotype (i.e. high vs. low) and an

158 additional selection marker encoded on a low copy number plasmid were co-incubated in liquid 159 LB medium containing herbicide, antibiotic, both, or neither and grown to saturation before 160 dilution by a factor of $10^{3}$ in the same conditions. The antibiotic concentration chosen was below 161 NOEL for both strains. NOEL is defined here as the highest antibiotic concentration for which 162 no effect on survival or selection were observed. The titer of each culture was determined by 163 plating on non-selective medium after each incubation step. After 5 rounds of incubation, the 164 ratio of strains was determined by selecting for the second - competition irrelevant - marker.

165 Natural selection was defined as the difference in the exponential growth rate of the two strains 166 (Mallet 2012; van den Bosch et al. 2014). Under this interpretation, the change in the proportion 167 of resistant individuals per unit of time is the logistic curve (Mallet 2012) with the explicit 168 solution $\mathrm{p}=\mathrm{e}^{\mathrm{st}} /\left(\mathrm{c}+\mathrm{e}^{\mathrm{st}}\right)$, where $\mathrm{t}=$ time, $\mathrm{p}=$ proportion of resistant individuals, $\mathrm{s}=$ strength of selection, $169 \mathrm{c}=$ constant describing the initial proportion of resistant individuals, $\mathrm{c}=\left(1 / \mathrm{p}_{0}\right)-1$. The constant $\mathrm{p}_{0}$ is 170 the proportion of resistant individuals at $t_{0}$ (the start of the experiment). $t$ is defined as the 171 number of generations, using the generation time for the more resistant strain. By rearranging 172 this formula, the strength of selection is $s=\ln (p c /(1-p)) / t$.

\section{Growth curves}

174 Growth curves were established at $37^{\circ} \mathrm{C}$ in liquid LB medium supplemented with herbicide, 175 antibiotic, both, or neither using a FLUOstar Omega microplate reader (BMG LABTECH, 176 Germany). The $\mathrm{OD}_{600}$ was determined every $6 \mathrm{~min}$ for $16 \mathrm{hrs}$ and averaged over five biological 
177 replicates. Cultures were started at densities of ca. $10^{6} \mathrm{cfu} / \mathrm{mL}$. Antibiotic and herbicide

178 concentrations used are detailed in the legend of Suppl. Fig. S1.

\section{Statistical analysis}

$180 \mathrm{R}$ was used for all statistical analyses (R Core Team 2013). For experiments measuring changes

181 to MIC, an ANOVA was used to analyze the randomized complete block design, using each

182 independent experiment as a block with presence/absence of antibiotic/herbicide as levels.

183 Residuals were used to test for normality and equality of variances and log transformed data

184 where appropriate. Tukey's HSD test was used to determine which treatments were significantly

185 different from each other.

186 For mixed culture experiments (changes to MSC), two sets of analyses were performed. First, we

187 determined whether adding antibiotics increases the strength of selection at different herbicide

188 concentrations. Second, we determined whether adding herbicides increases the strength of

189 selection at different antibiotic concentrations. Each set of questions was tested using contrasts

190 performed with the glht function in the multcomp package (Hothorn et al. 2008) with a two-sided

191 alternative and sequential Bonferroni procedure. These contrasts were fit to an ANOVA model

192 treating each combination of herbicide and antibiotic as a treatment category. Residual plots

193 confirmed that the assumptions of normality and equality of variance were met. Because

194 residuals were approximately normally distributed we report the arithmetic mean (the maximum

195 likelihood estimator of population mean under the assumption of normality).

196 Statistical analysis for growth curves

197 To estimate carrying capacity (k), a logistic growth model on the raw data using non-linear least

198 squares (nls) was fit in R. Differences in population growth between strains in different

199 treatments were estimated by estimating $r$, the intrinsic growth rate, of each strain. Data sets were

$200 \log$ transformed and the slope of the growth curve between $\mathrm{t}=48$ mins and $\mathrm{t}=150$ mins was

201 measured. A visual inspection of the plots revealed that before $\mathrm{t}=48 \mathrm{mins}$ graphs were not linear.

202 After $\mathrm{t}=150$ mins, growth slowed as cells were entering stationary phase.

203 We tested for differences between the two strains in both $\mathrm{r}$ and $\mathrm{k}$ by calculating contrast in an

204 ANOVA (aov). Residual plots were used to test for violations of assumptions for ANOVA.

205 Assumptions of normality and equal variances were met in all data sets. In two data sets there 
206 were a small number of outliers. These had small influences on parameter estimates and were

207 hence not removed. Contrasts between treatments were calculated using the glht package in $\mathrm{R}$ 208 using the sequential Bonferroni correction.

209

\section{Results}

211 As reported previously, simultaneous exposure to a variety of antibiotics and either various 212 commercial herbicide formulations (Kurenbach et al. 2015) or to either active ingredients and 213 some surfactants (Kurenbach et al. 2017), alters the survival of E. coli and S. typhimurium 214 compared to exposure to only the antibiotics. This was due to adaptive changes in exposed 215 bacteria. From these observations we hypothesized that particular combinations of antibiotics 216 and these formulations alter the MSC or MIC of the antibiotic in a way that favours genetically 217 resistant variants. To test this hypothesis, we conducted short-term evolution in antibiotics with 218 and without additional exposures to commercial herbicide formulations.

219 Because the effect of the herbicide exposure was specific to the combination of herbicide and 220 antibiotic, primarily two kinds of experiments were done. Firstly, in the case where the herbicide 221 raised the MIC of the antibiotic, we tested whether antibiotic survivors could give rise to 222 antibiotic resistant variants. In this case, only bacteria simultaneously exposed to both the 223 herbicide and antibiotic would survive when the antibiotic concentration exceeded the MIC of 224 the bacteria measured without the addition of the herbicide. Secondly, in the case where the 225 herbicide decreased the MIC of the antibiotic, we tested whether the herbicide decreased the 226 MSC of the antibiotic, leading to a greater rate of resistance evolution at lower concentrations of 227 antibiotic because of exposure to the herbicide formulation.

228

229 Evolution of acquired antibiotic resistance in cultures with herbicide-induced increases in 230 MIC

231 Exposing either E. coli or S. typhimurium to the herbicide formulation Roundup increased the 232 MIC of the fluoroquinolone antibiotic ciprofloxacin, as did exposing S. typhimurium to the 233 herbicide formulation Kamba (Kurenbach et al. 2015). These three combinations were used to 234 test the hypothesis that the survival of bacteria exposed to normally lethal concentrations of 
235 antibiotic provides the opportunity for the population to evolve higher frequencies of resistant 236 genotypes.

237 The rate of acquired resistance mutations in populations of E. coli BW25113 or S. typhimurium 238 SL3770 (all strains described in Table 1) was measured over the course of about 25 generations 239 in a standard rich growth medium, liquid LB, or LB+herbicide, LB+Cip, or LB+herbicide+Cip.

240 The chosen herbicide concentration was below NOEL (no observable effect level), so had no 241 impact on survival or selection of the bacteria on its own (Table 2). The ciprofloxacin

242 concentration was the same in all cultures to which it was added and was above MIC in cultures 243 not exposed to herbicide, but below MIC for bacteria in cultures simultaneously exposed to 244 herbicide. At the end of the experiment, bacteria were transferred to solid medium supplemented 245 with high levels of ciprofloxacin (above MIC) and no herbicide, which was permissive only to 246 the growth of variants with acquired resistance.

247 Expectedly, cultures supplemented only with ciprofloxacin did not grow, and genotypic resistant 248 variants were not detected. Cultures that grew for 25 generations without ciprofloxacin 249 supplementation produced resistant variants at similar low rates regardless of exposure to the 250 herbicide formulations. This indicated that the herbicides were not mutagens at these 251 concentrations. In a separate standard test of mutagenicity (Funchain et al. 2001), bacteria were 252 exposed to herbicides and plated on the antibiotic rifampicin. No difference in resistance rates 253 was observed $(\mathrm{p}=0.3873)$.

254 Populations of bacteria with continuous exposure to herbicide and antibiotic had significantly 255 higher numbers of ciprofloxacin resistant variants. The rate ranged from $10^{2}$ times higher for the 256 combination of Cip $+\mathrm{Kamba}+$ S. typhimurium to $10^{5}$ times higher for Cip+Roundup $+E$. coli

257 (Table 2). This is consistent with our prediction that herbicide-induced adaptive resistance that 258 increases MIC allows rare spontaneously arising ciprofloxacin resistant mutants to increase a 259 culture's MIC. Because ciprofloxacin is mutagenic (Cirz et al. 2005), it contributes quantitatively 260 to the genotypic heterogeneity, including acquired antibiotic resistance, among the bacteria that 261 survive because of the effects of the herbicide.

262 Ciprofloxacin resistant colonies were isolated at the end of each experiment from all treatments.

263 We determined MICs for 56 S. typhimurium isolates (isolated on $0.05 \mu \mathrm{g} / \mathrm{mL}$ Cip), 27 isolates

264 from Kamba+Cip and 29 isolates from Roundup+Cip treatments. The parental strain and 2 
265 isolates from each LB, Kamba, and Roundup treatments, also isolated on $0.05 \mu \mathrm{g} / \mathrm{mL}$ Cip, were 266 included as controls.

267 The parental strain and 6 evolved isolates did not grow at $0.07 \mu \mathrm{g} / \mathrm{mL}$, a concentration just above 268 the selection concentration. We observed MICs of $0.1 \mu \mathrm{g} / \mathrm{mL}$ ciprofloxacin for 21 isolates and $2690.2 \mu \mathrm{g} / \mathrm{mL}$ ciprofloxacin for 28 isolates. Only 7 isolates displayed higher MICs (2 from 270 Kamba+Cip, 4 from Roundup+Cip, and 1 from LB), the highest being $1.25 \mu \mathrm{g} / \mathrm{mL}$ ciprofloxacin

271 reached by one isolate recovered from a Roundup+Cip culture. We observed no correlation

272 between level of resistance and original treatment, which indicates that there are no qualitative 273 differences between the ciprofloxacin resistant variants arising in the different treatments.

274

275 Evolution of acquired antibiotic resistance in cultures with herbicide-induced decreases in 276 MIC

277 Antibiotic resistant bacteria are becoming a fixed part of many environments despite the 278 concentration of antibiotics often being very low (Hermsen et al. 2012). We hypothesized that 279 exposure to some herbicides can shift the MSC to lower antibiotic concentrations leading to 280 competition between individuals with different physiological responses and thus providing an

281 environment in which genotypically resistant bacteria evolve.

282 Mixed cultures of E. coli were created to represent pre-existing antibiotic resistance 283 heterogeneity within natural environments. The phenotypic differences were created using

284 bacteria carrying isogenic plasmids with different alleles of tet $A$ (Tet ${ }^{\text {high }}$, Tet ${ }^{\text {low }}$, Table 1$)$. The MIC for tetracycline of Tet ${ }^{\text {low }}$ was $1.5 \mu \mathrm{g} / \mathrm{mL}$ and for Tet ${ }^{\text {high }}$ it was $125 \mu \mathrm{g} / \mathrm{mL}$.

286 Tet ${ }^{\text {high }}$ and Tet $^{\text {low }}$ were competed in liquid LB, LB+Tet, and LB+Tet+Roundup media for about 28742 generations. This herbicide lowers the antibiotic MIC (Fig. 1B). The same concentration of 288 Roundup was used in combination with various concentrations of tetracycline, all of which were 289 below the MIC of Tet ${ }^{\text {low }}$. Roundup reduced tetracycline MIC for Tet ${ }^{\text {low }}$ to $0.5 \mu \mathrm{g} / \mathrm{mL}$, but growth 290 of Tet ${ }^{\text {high }}$ was not affected for tetracycline concentrations up to $50 \mu \mathrm{g} / \mathrm{ml}$, far above levels used in 291 this experiment.

292 The MSC, i.e. the concentration of tetracycline at which one strain started to dominate the 293 culture, was determined from the calculated selection coefficients. A positive coefficient 
294 indicated selection for the more resistant strain. The selection coefficient was significantly 295 different for competitions in environments supplemented with $\geq 0.1 \mu \mathrm{g} / \mathrm{mL}$ tetracycline compared 296 to competitions in LB medium (Fig. 2). Mixed cultures in LB+Tet+Roundup had significantly 297 different selection coefficients compared to the LB competitions beginning at a tetracycline 298 concentration of $0.05 \mu \mathrm{g} / \mathrm{mL}$.

299 A reverse experiment was performed using Kamba instead of Roundup. This herbicide increases 300 the MIC of Tet ${ }^{\text {tow }}$ from 1.5 to $3 \mu \mathrm{g} / \mathrm{mL}$. When Kamba was part of the environment, the selection 301 coefficient was significantly different from the competition in LB medium at a tetracycline 302 concentration $\geq 0.5 \mu \mathrm{g} / \mathrm{mL}$, compared to $\geq 0.1 \mu \mathrm{g} / \mathrm{mL}$ without Kamba (Fig. 2). In both cases, MIC 303 for the Tet ${ }^{\text {tow }}$ strain and MSC moved in the same direction.

305 Herbicide-induced changes in MSC can occur without a change in MIC

306 In addition to the observed increase or decrease of antibiotic MIC caused by herbicide

307 formulations, we also observed combinations of antibiotic and herbicide that did not change the 308 MIC of the antibiotic but did alter growth rate (Fig. 1C). For example, E. coli cultures in medium 309 supplemented with Kamba grew faster at some sub-lethal concentrations of ciprofloxacin, but 310 Kamba did not change the MIC (Kurenbach et al. 2015). At the concentrations of the antibiotic at 311 which the herbicide altered growth rate, possibly also the MSC of the antibiotic was changed.

312 To test this, we measured the frequency at which acquired ciprofloxacin resistance arose during 313 culture in the combination of Cip+Kamba $+E$. coli. The mutation rate of an E. coli monoculture 314 was measured over the course of about 25 generations in a standard rich growth medium, liquid 315 LB, and in LB supplemented with Cip, Kamba, or Cip+Kamba. The experiment was similar to 316 those described in the section "Evolution of acquired antibiotic resistance in cultures with 317 herbicide-induced increases in MIC", but here the ciprofloxacin concentration was below MIC 318 and high enough to slow the growth of bacteria in cultures not supplemented with herbicide. At 319 the end of the experiment, bacteria were transferred to solid medium supplemented with 320 ciprofloxacin at $>$ MIC and no herbicide, which was permissive only to the growth of variants 321 with acquired resistance. 
322 Acquired resistance rates were the same for cultures grown in LB, LB+Kamba or

$323 \mathrm{LB}+\mathrm{Cip}+\mathrm{Kamba}$ (for both treatment combinations $\mathrm{p}>0.2$; Table 3 ). The acquired resistance rate

324 in the LB+Cip culture was about $10^{5}$ times higher than the other three cultures $\left(\mathrm{p}<10^{-4}\right.$ for all

325 treatment combinations), indicating that the ciprofloxacin concentration met or exceeded the

326 MSC. The addition of Kamba neutralized the selective effect of ciprofloxacin by shifting the

327 MSC to a higher level without changing the MIC.

\section{Herbicide-induced changes to MSC are herbicide concentration-dependent}

330 Pairs of isogenic strains of E. coli (Table 1) with either different streptomycin (Str ${ }^{\text {high }}$, Str ${ }^{\text {low }}$ ) or 331 tetracycline (Tet ${ }^{\text {high }}$, Tet ${ }^{\text {low }}$ ) MICs were competed for about 40 generations. The frequency of 332 each strain after competition was measured. The concentration of the appropriate antibiotic did 333 not vary between cultures, but the concentration of herbicide did. Combinations were chosen that 334 lead to a decrease in MIC with the chosen herbicide, namely Str+Kamba, and Tet+Roundup.

335 There was no differential in the fitness of paired strains when they were co-cultured in standard 336 rich medium (Fig. 3). In cultures containing antibiotic but not supplemented with herbicide

337 neither strain had a fitness advantage, indicating that the antibiotic concentration was below 338 MSC. Likewise, the herbicide alone did not affect fitness except for a statistically non-significant 339 effect at the highest tested concentration of Roundup.

340 In each test, the combination of antibiotic and herbicide reduced MSC. The more resistant strain 341 invariably increased relative to the isogenic competitor in a herbicide dose-dependent manner 342 when both antibiotic and herbicide were in the environment. Selection coefficients were 343 statistically significantly different from LB medium at 183 parts per million acid equivalent 344 (ppm ae) Kamba and 311 ppm ae Roundup.

345 The observed fitness differential was due to the faster reproductive rate of the Strhigh and Tet ${ }^{\text {high }}$ 346 strains in environments with sub-lethal concentrations of antibiotic for both strains in each pair.

347 This was shown by measuring the growth rate of the strains in monoculture rather than in 348 competition (Supplementary Fig. S1). No differences were observed for the monoculture growth 349 rate ( $r$ ) of matched isogenic pairs in LB medium, $L B+$ antibiotic, or LB+herbicide ( $\mathrm{p}>0.1$ for all 350 combinations; Supplementary Fig. S1A and C). Only in the combined LB+herbicide+antibiotic 
351 treatment did strains with higher MICs have shorter generation times in monoculture than their

352 lower MIC counterparts $\left(\mathrm{p}=1.6 \times 10^{-5}\right.$ (Kamba+Str) and $\mathrm{p}=4.7 \times 10^{-10}$ (Roundup+Tet)).

353 Significant differences in the carrying capacity of the environments for all treatment

354 combinations were observed for the matched strains exposed to Kamba+Str measured in

355 monoculture. The high MIC strain achieved higher final optical densities $\left(\mathrm{p}<2 \times 10^{-16}\right.$;

356 Supplementary Fig. S1B). In contrast, the Strow strain population grew to higher final optical

357 densities in LB, LB+Str, and LB+Kamba treatments. In cultures with Roundup+Tet, Tet ${ }^{\text {high }}$ and

358 Tet ${ }^{\text {low }}$ had similar densities $(\mathrm{p}=0.24$, Supplementary Fig. S1D).

359

360 Discussion

361 In this study we report that when bacteria are simultaneously exposed to herbicides and

362 antibiotics, mutants with higher levels of resistance can evolve. In some cases, resistance evolved

363100,000 times faster.

364 Herbicides can increase the MIC of some antibiotics. At what otherwise would be a lethal

365 concentration of the antibiotic, the bacteria can continue to reproduce. Each reproductive event

366 has a low but steady potential of producing a variant daughter with a higher MIC. We found that

367 these strains have a fitness advantage and accumulate differentially to their low MIC cousins.

368 Herbicides can also decrease the MIC of some antibiotics. At what otherwise would be a

369 concentration of antibiotic below the MSC, too low to have an effect on the fitness of two

370 bacteria differing in their MICs, we found that the bacteria with the higher MICs replaced the

371 bacteria with lower MICs. The shift in MSC seemed to be of a similar magnitude as the shift in

372 MIC observed for the lower MIC strain.

373 Finally, herbicides can also alter survival potential at some antibiotic concentrations but not

374 change the concentration to which the entire population is innately susceptible. In the case

375 presented here, MSC was shifted by the herbicides, but MIC was not. The herbicide mitigated

376 the selective pressure caused by the antibiotic, and no genotypes with higher resistance levels

377 established in the population. Although not tested, it is likely that in the reverse case, when MSC

378 is lowered by a substance, antibiotic resistance may arise at higher frequencies. 
379 Our research shows that manufactured chemical products such as the herbicides can have a 380 complex effect on the evolution of antibiotic resistance. They did not replace antibiotics, but 381 could accelerate resistance evolution. Herbicides that reduce the MSC will be more effective at 382 stimulating resistance evolution at the lower ends of the antibiotic concentration gradient, while 383 herbicides that increase the MIC will be more effective at stimulating resistance evolution at the 384 higher ends.

385 Antibiotics can influence acquired resistance evolution in a number of ways. First, some 386 antibiotics may also be mutagens (Cirz et al. 2005) and at sub-lethal concentrations could 387 increase the probability of a mutation conferring resistance arising in the population. Second, 388 they discriminate between the reproductive rate of those with higher and lower tolerances to the 389 toxic effects of the antibiotic, leading to different frequencies of genotypes that are less 390 susceptible. At the environmentally relevant concentrations of herbicide formulations that we 391 used, no mutagenic activity was detected. However, the herbicides did contribute to the ability of 392 antibiotics to change the frequency of resistant variants in populations.

393 Combination treatments using a non-antibiotic to increase an antibiotic's effectiveness have been 394 suggested to help preserve the usefulness of antibiotics (Allen et al. 2014; Schneider et al. 2017; 395 Wright 2016). Indeed, various combinations are in clinical use (Worthington \& Melander 2013). 396 While this might improve or enable treatment of an infection, our results indicate that decreasing 397 bacteria's survival by making an antibiotic more potent may promote resistance evolution by 398 creating more environments in which a resistance genotype is more fit.

399 Herbicides and different ingredients in product formulations can have antimicrobial activities, 400 with some being more potent antimicrobial agents than are others (Kurenbach et al. 2017). For 401 example, Roundup was more toxic than Kamba to the bacteria that we used. The low level 402 toxicity is likely how they induce adaptive resistance, a source of phenotypic heterogeneity 403 (Kurenbach et al. 2015). In addition, biocidal agents that are mutagens may introduce genotypic 404 heterogeneity.

405 Antibiotic and herbicide gradients may be viewed as two environmental dimensions 406 concentrating competition between bacteria of different genotypes and physiotypes. The 407 particular concentrations of antibiotic and herbicide at any point of intersection of the gradients 408 above a threshold level increases the frequency of the most fit strains and species, amplifying 
409 any linked or associated traits in the more fit competitor. While antibiotics can do this without 410 herbicides (Andersson \& Hughes 2014; Baquero et al. 1998a; Denamur \& Matic 2006), the

411 herbicides used in this study increased the range of concentrations under which the antibiotic 412 affects the evolution of resistance.

413 The concentrations of herbicides we used were below recommended application levels and hence 414 within relevant environmental ranges, suggesting that what we observe in the laboratory has the 415 potential to occur in many places where both antibiotics and herbicides are found together.

416 Simultaneous herbicide and antibiotic exposures are common. Herbicides are used in agriculture, 417 where spray drift or walking through treated fields exposes farm livestock and pets, which may 418 be on therapeutic or prophylactic antibiotics. Most ingested antibiotic is not metabolized and thus 419 excreted (Chee-Sanford et al. 2009), becoming mixed with soil as crop fertilizer which in situ 420 may be subsequently sprayed with herbicide. Microbes from these mixes may be carried by 421 blow- and house-flies (Zurek \& Ghosh 2014). Likewise honeybees may be exposed to herbicide 422 spray or residues as they forage and return to an antibiotic-treated hive. Additionally, herbicides 423 are used in urban environments for purposes like gardening and lawn care, including parks and 424 roadsides (Atwood \& Paisley-Jones 2017). Worldwide, herbicide use was approximately $1 \times 10^{9}$ 425 kilograms in 2012 with up to $2 \times 10^{8}$ kilograms of the active herbicidal ingredients glyphosate, 2, 426 4-D and dicamba used in the US in 2012 (Atwood \& Paisley-Jones 2017).

427 Other chemicals also have been shown to cause adaptive resistance and to increase resistance 428 frequencies (Egeghy et al. 2012; Gustafson et al. 1999; Levy 2001). Non-antibiotic prescription 429 medicines and food emulsifiers select antibiotic resistant gut bacteria (Kurenbach et al. 2017; 430 Maier et al. 2018). Approximately 8 million manufactured chemical substances are currently in 431 commerce (Egeghy et al. 2012; Shen et al. 2011). According to the US Environmental Protection 432 Agency, annual production of each of the top 3,000 chemicals is greater than $6 \times 10^{11}$ 433 kilograms/year (EPA 2008). They are not regulated for effects on antibiotic resistance and not 434 tested for such effects.

435 The susceptibility of bacteria to antibiotics must be seen as a non-renewable resource, one that 436 requires careful stewardship worldwide (Amabile-Cuevas 2016; Heinemann \& Kurenbach 2017). 437 Evidence that antibiotic resistance evolution is influenced by exposure of bacteria to a wide 438 range of substances may require us to make changes in how we manage both antibiotics and 
439 other manufactured and widely distributed chemical products. This is because many facets of the 440 extrinsic environment induce adaptive changes, a complexity frequently ignored in standard 441 studies of resistance. As our results show, complex effects of exposures to non-therapeutic 442 chemicals may undermine strategies to preserve the effectiveness of antibiotics through altering

443 just their use. To our knowledge, there has been no attempt to systematically test common

444 chemicals to which pathogenic bacteria are chronically exposed for effects on antibiotic 445 resistance.

\section{Conclusions}

447 Neither reducing the use of antibiotics nor discovery of new ones may prevent the post-antibiotic 448 era. This is because bacteria may be exposed to other non-antibiotic chemicals that predispose 449 them to evolve resistance to antibiotics more quickly. Herbicides are examples of some of the 450 most common non-antibiotic formulations in frequent global use. More research is necessary to 451 see to what extend other different manufactured chemicals may contribute to this effect.

452 Moreover, depending on how the manufactured chemicals are used, or how they move through 453 the waste stream, there may be combinatorial effects caused by mixtures of different products.

454 Future work should take into account likely combinations as well as different ways that microbes 455 could be exposed to chemical products.

\section{Acknowledgements}

457 The authors thank Mark Silby for helpful comments. 
459

460

461

462

463

464

465

466

467

468

469

470

471

472

473

474

475

476

477

478

479

480

481

482

483

484

485

486

487

488

489

490

491

492

493

494

495

\section{References}

Allen HK, Trachsel J, Looft T, and Casey TA. 2014. Finding alternatives to antibiotics. Annals of the New York Academy of Sciences 1323:91-100. 10.1111/nyas.12468

Amabile-Cuevas CF. 2016. Society must seize control of the antibiotics crisis. Nature 533:439. 10.1038/533439a

Andersson DI, and Hughes D. 2014. Microbiological effects of sublethal levels of antibiotics. Nature Reviews Microbiology 12:465-478. 10.1038/nrmicro3270

Atwood D, and Paisley-Jones C. 2017. Pesticide industry sales and usage 2008-2012 market estimates. United States Environmental Protection Agency.

Baba T, Ara T, Hasegawa M, Takai Y, Okumura Y, Baba M, Datsenko KA, Tomita M, Wanner BL, and Mori H. 2006. Construction of Escherichia coli K-12 in-frame, single-gene knockout mutants: the Keio collection. Molecular Systems Biology 2:2006 0008. $10.1038 / \mathrm{msb} 4100050$

Baquero F, Negri MC, Morosini MI, and Blazquez J. 1998a. Antibiotic-selective environments. Clinical Infactious Diseases 27 Suppl 1:S5-11.

Baquero F, Negri MC, Morosini MI, and Blazquez J. 1998b. Selection of very small differences in bacterial evolution. International Microbiology 1:295-300.

Blair JM, Webber MA, Baylay AJ, Ogbolu DO, and Piddock LJ. 2015. Molecular mechanisms of antibiotic resistance. Nature Reviews Microbiology 13:42-51. 10.1038/nrmicro3380

CDC. 2013. Antibiotic resistance threats in the United States, 2013. Atlanta, GA, USA: Centers for Disease Control and Prevention.

Chee-Sanford JC, Mackie RI, Koike S, Krapac IG, Lin YF, Yannarell AC, Maxwell S, and Aminov RI. 2009. Fate and transport of antibiotic residues and antibiotic resistance genes following land application of manure waste. Journal of Environmental Quality 38:1086-1108. 10.2134/jeq2008.0128

Cirz RT, Chin JK, Andes DR, de Crecy-Lagard V, Craig WA, and Romesberg FE. 2005. Inhibition of mutation and combating the evolution of antibiotic resistance. PLoS Biology 3:e176. 10.1371/journal.pbio.0030176

Cohen SP, McMurry LM, Hooper DC, Wolfson JS, and Levy SB. 1989. Cross-resistance to fluoroquinolones in multiple-antibiotic-resistant (Mar) Escherichia coli selected by tetracycline or chloramphenicol: decreased drug accumulation associated with membrane changes in addition to OmpF reduction. Antimicrobial Agents and Chemotherapy 33:1318-1325.

Collingnon PC, Conly JM, Andremont A, and McEwen SA. 2016. World Health Organization ranking of antimicrobials according to their importance in human medicine: a critical step for developing risk management strategies to control antimicrobial 
496

497

498

499

500

501

502

503

504

505

506

507

508

509

510

511

512

513

514

515

516

517

518

519

520

521

522

523

524

525

526

527

528

529

530

531

532

533

534

535

resistance from food and animal production. Clinical Infactious Diseases 63:10871093.

Dan S, Shah A, Justo JA, Bookstaver PB, Kohn J, Albrecht H, and Al-Hasan MN. 2016. Prediction of Fluoroquinolone Resistance in Gram-Negative Bacteria Causing Bloodstream Infections. Antimicrobial Agents and Chemotherapy 60:2265-2272. 10.1128/AAC.02728-15

Denamur E, and Matic I. 2006. Evolution of mutation rates in bacteria. Molecular Microbiology 60:820-827.10.1111/j.1365-2958.2006.05150.x

Egeghy PP, Judson R, Gangwal S, Mosher S, Smith D, Vail J, and Cohen Ubal EA. 2012. The exposure data landscape for manufactured chemicals. Science of The Total Environment 414:159-166.

EPA. 2008. 2006 Inventory Update Reporting: Data Summary. In: Agency UEP, editor: US Environmental Protection Agency.

Fernandez L, Breidenstein EB, and Hancock RE. 2011. Creeping baselines and adaptive resistance to antibiotics. Drug Resistance Updates 14:1-21. 10.1016/j.drup.2011.01.001

Fernandez L, and Hancock RE. 2012. Adaptive and mutational resistance: role of porins and efflux pumps in drug resistance. Clinical Microbiology Reviews 25:661-681. 10.1128/CMR.00043-12

Funchain P, Yeung A, Stewart J, Clendenin WM, and Miller JH. 2001. Amplification of mutator cells in a population as a result of horizontal gene transfer. Journal of Bacteriology 183:3737-3741.

Gustafson JE, Candelaria PV, Fisher SA, Goodridge JP, Lichocik TM, McWilliams TM, Price CT, O'Brien FG, and Grubb WB. 1999. Growth in the presence of salicylate increases fluoroquinolone resistance in Staphylococcus aureus. Antimicrobial Agents and Chemotherapy 43:990-992.

Heinemann JA, and Kurenbach B. 2017. Post-Antibiotic Era is Business as Usual. Alliance for the prudent use of antibiotics (APUA) Newsletter 35:7-9.

Heinemann JA, Scott HE, and Williams M. 1996. Doing the conjugative two-step: evidence of recipient autonomy in retrotransfer. Genetics 143:1425-1435.

Heinemann JA, and Sprague GF, Jr. 1989. Bacterial conjugative plasmids mobilize DNA transfer between bacteria and yeast. Nature 340:205-209. 10.1038/340205a0

Hermsen R, Deris JB, and Hwa T. 2012. On the rapidity of antibiotic resistance evolution facilitated by a concentration gradient. Proceedings of the National Academy of Sciences 109:10775-10780. 10.1073/pnas.1117716109

Hothorn T, Bretz F, and Westfall P. 2008. Simultaneous inference in general parametric models. Biometrical Journal 50:346-363. 10.1002/bimj.200810425

Kurenbach B, Gibson PS, Hill AM, Bitzer AS, Silby MW, Godsoe W, and Heinemann JA. 2017. Herbicide ingredients change Salmonella enterica sv. Typhimurium and Escherichia coli antibiotic responses. Microbiology 163:1791-1801. 10.1099/mic.0.000573 
536 Kurenbach B, Marjoshi D, Amabile-Cuevas CF, Ferguson GC, Godsoe W, Gibson P, and

537

538

539

540

541

542

543

544

545

546

547

548

549

550

551

552

553

554

555

556

557

558

559

560

561

562

563

564

565

566

567

568

569

570

571

572

573

574

575

Heinemann JA. 2015. Sublethal exposure to commercial formulations of the herbicides dicamba, 2,4-dichlorophenoxyacetic acid, and glyphosate cause changes in antibiotic susceptibility in Escherichia coli and Salmonella enterica serovar Typhimurium. mBio 6. 10.1128/mBio.00009-15

Levy SB. 2001. Antibacterial household products: cause for concern. Emerging Infectious Diseases 7:512-515. 10.3201/eid0707.010705

Maier L, Pruteanu M, Kuhn M, Zeller G, Telzerow A, Anderson EE, Brochado AR, Fernandez KC, Dose H, Mori H, Patil KR, Bork P, and Typas A. 2018. Extensive impact of nonantibiotic drugs on human gut bacteria. Nature 555:623-628. 10.1038/nature25979

Mallet J. 2012. The struggle for existence: how the notion of carrying capacity, K, obscures the links between demography, Darwinian evolution, and speciation. Evolutionary Ecology Research 14:627-665.

Palmer AC, and Kishony R. 2013. Understanding, predicting and manipulating the genotypic evolution of antibiotic resistance. Nature Reviews Genetics 14:243-248. $10.1038 / \operatorname{nrg} 3351$

R Core Team. 2013. R: a language and environment for statistical computing. Vienna, Austria: R Foundation for Statistical Computing.

Roantree RJ, Kuo TT, and MacPhee DG. 1977. The effect of defined lipopolysaccharide core defects upon antibiotic resistances of Salmonella typhimurium. Journal of General Microbiology 103:223-234.

Sanchez-Romero MA, and Casadesus J. 2014. Contribution of phenotypic heterogeneity to adaptive antibiotic resistance. Proceedings of the National Academy of Sciences 111:355-360. 10.1073/pnas.1316084111

Schneider EK, Reyes-Ortega F, Velkov T, and Li J. 2017. Antibiotic-non-antibiotic combinations for combating extremely drug-resistant Gram-negative 'superbugs'. Essays in Biochemistry 61:115-125. 10.1042/EBC20160058

Scholz P, Haring V, Wittmann-Liebold B, Ashman K, Bagdasarian M, and Scherzinger E. 1989. Complete nucleotide sequence and gene organization of the broad-host-range plasmid RSF1010. Gene 75:271-288.

Shen Z, Pu XY, and Zhang Q. 2011. Salicylate functions as an efflux pump inducer and promotes the emergence of fluoroquinolone-resistant Campylobacter jejuni mutants. Applied and Environmental Microbiology 77:7128-7133. 10.1128/AEM.00763-11

Stockwell VO, and Duffy B. 2013. Use of antibiotics in plant agriculture. Scientific and Technical Review of the Office International des Epizooties 31:199-210.

Sutcliffe JG. 1979. Complete nucleotide sequence of the Escherichia coli plasmid pBR322. Cold Spring Harbour Symposia on Quantitative Biology 43 Pt 1:77-90.

Teillant A, Gandra S, Barter D, Morgan DJ, and Laxminarayan R. 2015. Potential burden of antibiotic resistance on surgery and cancer chemotherapy antibiotic prophylaxis in 
576

577

578

579

580

581

582

583

584

585

586

587

588

589

590

591

592

593

594

595

596

597 the USA: a literature review and modelling study. Lancet Infectious Diseases 15:1429-1437.

Thomas MG, Smith AJ, and Tilyard M. 2014. Rising antimicrobial resistance: a strong reason to reduce excessive antimicrobial consumption in New Zealand. New Zealand Medical Journal 127:72-84.

Van Boeckel TP, Brower C, Gilbert M, Grenfell BT, Levin SA, Robinson TP, Teillant A, and Laxminarayan R. 2015. Global trends in antimicrobial use in food animals. Proceedings of the National Academy of Sciences 112:5649-5654. $10.1073 /$ pnas. 1503141112

van den Bosch F, Oliver R, van den Berg F, and Paveley N. 2014. Governing principles can guide fungicide-resistance management tactics. Annual Reviews Phytopathology 52:175-195. 10.1146/annurev-phyto-102313-050158

Worthington RJ, and Melander C. 2013. Combination approaches to combat multidrugresistant bacteria. Trends in Biotechnology 31:177-184. 10.1016/j.tibtech.2012.12.006

Wright GD. 2016. Antibiotic Adjuvants: Rescuing Antibiotics from Resistance. Trends in Microbiology 24:862-871. 10.1016/j.tim.2016.06.009

Zurek L, and Ghosh A. 2014. Insects represent a link between food animal farms and the urban environment for antibiotic resistance traits. Applied Environmental Microbiology 80:3562-3567. 10.1128/AEM.00600-14 


\section{Figure 1 (on next page)}

Effects of herbicides on bacterial responses to antibiotics.

Herbicides can change responses to antibiotics. Solid curves indicate survival as a function of only antibiotic concentration, intersecting the x-axes at the MIC. Dashed curves show herbicides (A) increasing the MIC, (B) decreasing the MIC, and (C) having no effect on MIC but altering population survival at antibiotic concentrations below-MIC. The hypothetical MSC is the point of divergence of solid and dashed curves and the area in gray illustrates all antibiotic concentrations where the herbicide changes the response to antibiotics. 


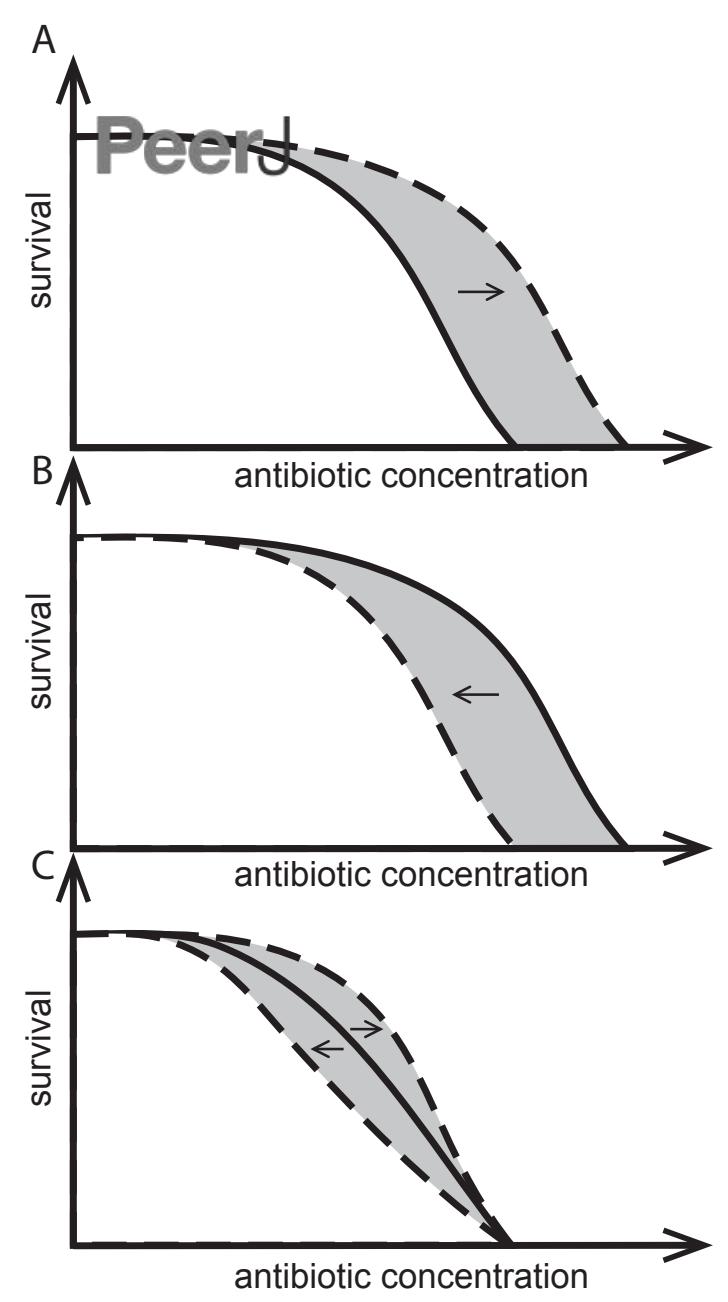




\section{Figure 2 (on next page)}

Competition between strains of different potential to resist tetracycline.

Competition between tetracycline resistant (AH201, Tet ${ }^{\text {high }}$ ) and susceptible (AH214, Tet ${ }^{\text {low }}$ )

strains of $E$. coli in different concentrations of tetracycline with or without Kamba $(K)$ or

Roundup (R). Kamba was used at 1830 ppm ae, Roundup at 311 ppm ae. Selection coefficients are presented as a function of tetracycline concentration, with positive values indicating a selection for the more resistant strain. Values are means of three independent experiments. To represent sampling uncertainty in the mean value for treatment groups we present error bars depicting standard errors (standard deviation $/ \sqrt{ } n$ ). * indicate the lowest tetracycline concentrations for each treatment where selection coefficients were significantly different from the -Tet/-herbicide treatment, based on a Bonferroni corrected contrast described in the Materials and Methods section. 


\section{Figure 3 (on next page)}

Dose-response to two herbicides with $(\mathbf{O})$ or without $(\mathbf{\Delta})$ antibiotic (used at a single concentration).

Dose-response of $E$. coli to two herbicides with $(\mathbf{O})$ or without $(\boldsymbol{\Delta})$ antibiotic (used at a single concentration). A positive selection coefficient indicates selection for the strain with a higher MIC. Results are averages of three independent experiments. To represent sampling uncertainty in the mean value for treatment groups we present error bars depicting standard errors (standard deviation/Vn). (A): Kamba+Str, using strains AH204 (Strigh, MIC $_{\text {Str: }}: 200 \mu \mathrm{g} / \mathrm{mL}$ ) and $\mathrm{AH} 211$ (Strlow, $\mathrm{MIC}_{\text {Stri }}: 1 \mu \mathrm{g} / \mathrm{mL}$ ); (B): Roundup+Tet, using strains AH201 (Tet ${ }^{\text {high }}$, MIC Tet $^{\text {: }}$ $125 \mu \mathrm{g} / \mathrm{mL}$ ) and $\mathrm{AH} 214$ (Tet ${ }^{\text {low }}, \mathrm{MIC}_{\text {Tet }}: 1.5 \mu \mathrm{g} / \mathrm{mL}$ ). Concentrations of antibiotics were $0.25 \mu \mathrm{g} / \mathrm{mL}$ for Str and $0.05 \mu \mathrm{g} / \mathrm{mL}$ for Tet. Asterisks indicate contrasts where addition of antibiotic significantly changed the strength of selection, based on a Bonferroni corrected contrast described in the Materials and Methods section, with *: $p<0.05$; **: $p<0.01$; ***: $p<0.001$. 


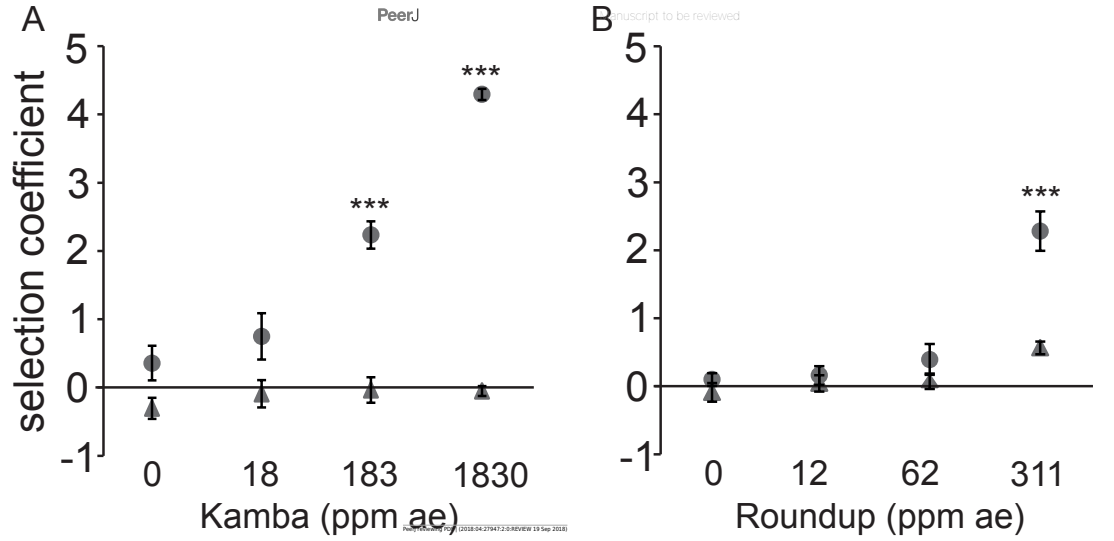




\section{Table 1 (on next page)}

Bacteria and plasmids

*Kamba: added at 1830 ppm ae, Roundup added at 1240 ppm ae. 


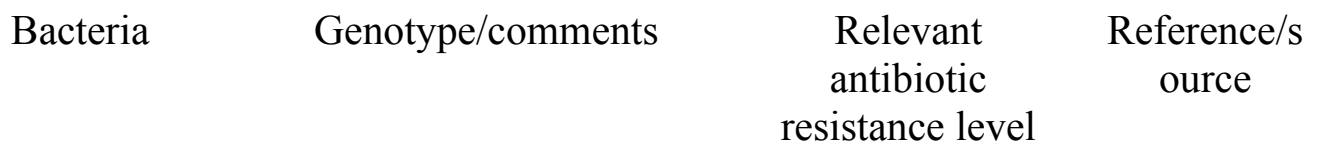

E. coli

BW25113

wild-type. $\mathrm{F}^{-}, \lambda-\Delta(\mathrm{araD}-$

Cip/Cip+Kamba/

(Baba et al. araB) 567, AlacZ4787(::rrnB-3), rph1, $\Delta$ (rhaD-rhaB) 568, Cip+Roundup*: 2006)

Solid medium: hsdR514

$0.01 / 0.07 / 0.07$

$\mu \mathrm{g} / \mathrm{mL}$

Liquid medium:

0.04/0.05/0.09

$\mu \mathrm{g} / \mathrm{mL}$

SB21 hsdS, leuB6, thr

(Heinemann

\& Sprague

1989)

AH201 (Tet $\left.{ }^{\text {high }}\right) \quad$ SB21 (pBR322)

Tet: $125 \mu \mathrm{g} / \mathrm{mL} \quad$ This study

AH214(Tet $\left.{ }^{\text {low }}\right) \quad$ SB21 (pAH14)

Tet: $1.5 \mu \mathrm{g} / \mathrm{mL} \quad$ This study

JB436

SB21 Nal ${ }^{\mathrm{R}}$

Nal: $60 \mu \mathrm{g} / \mathrm{mL}$

(Heinemann et al. 1996)

AH204 (Str $\left.{ }^{\text {high}}\right) \quad$ JB436 (RSF1010)

Str: $250 \mu \mathrm{g} / \mathrm{mL}$

This study

AH211 (Strow) SB21 (pAH11)

Str: $1 \mu \mathrm{g} / \mathrm{mL}$

This study

S. enterica sv Typhimurium

SL3770

$$
\mathrm{LT} 2, p y r^{+}, r f a
$$

Cip/Cip+Kamba/ (Roantree et Cip+Roundup*:

al. 1977)

Solid medium:

$0.035 / 0.1 / 0.1$

$\mu \mathrm{g} / \mathrm{mL}$

Liquid medium:

0.05/0.1/0.2

$\mu \mathrm{g} / \mathrm{mL}$

Plasmids

\begin{tabular}{|c|c|c|c|}
\hline pBR322 & $\mathrm{Amp}^{\mathrm{R}}, \mathrm{Tet}^{\mathrm{R}}$ & $\begin{array}{l}\text { Amp: } 100 \mu \mathrm{g} / \mathrm{mL} \text {, } \\
\text { Tet: } 125 \mu \mathrm{g} / \mathrm{mL}\end{array}$ & $\begin{array}{r}\text { (Sutcliffe } \\
1979)\end{array}$ \\
\hline pAH14 & $\begin{array}{l}\text { pBR322 derivative, } \text { Cam }^{\mathrm{R}} \text {, } \\
\text { Amp }^{\mathrm{S}}, \mathrm{Tet}^{\mathrm{S}}\end{array}$ & Cam:20 $\mu \mathrm{g} / \mathrm{mL}$ & This study \\
\hline RSF1010 & $\mathrm{Str}^{\mathrm{R}}$ & Str: $250 \mu \mathrm{g} / \mathrm{mL}$ & $\begin{array}{r}\text { (Scholz et } \\
\text { al. 1989) }\end{array}$ \\
\hline
\end{tabular}




\begin{tabular}{|c|c|c|c|}
\hline pAH11 & $\begin{array}{l}\text { RSF1010 derivative, } \\
\text { Cam }^{\mathrm{R}}, \mathrm{Str}^{\mathrm{S}}\end{array}$ & $\begin{array}{l}\text { Cam: } 20 \mu \mathrm{g} / \mathrm{mL} \text {, } \\
\text { Str: } 1 \mu \mathrm{g} / \mathrm{mL}\end{array}$ & This study \\
\hline
\end{tabular}

1 


\section{Table 2 (on next page)}

Mutation rate (mutant frequency per generation)

Rates of acquired resistance were determined with or without herbicide and Cip exposure. Values are means of at least four independent experiments. To represent sampling uncertainty in the mean value for treatment groups we present standard errors (standard deviation $/ \sqrt{ } \mathrm{n}$ ) in brackets. Cip concentrations used were $0.07 \mu \mathrm{g} / \mathrm{mL}$ for S. enterica and 0.05 $\mu \mathrm{g} / \mathrm{mL}$ for S. enterica in liquid culture and : $0.07 \mu \mathrm{g} / \mathrm{mL}$ for S. enterica and $0.06 \mu \mathrm{g} / \mathrm{mL}$ for $E$. coli for final plating. 1,250 ppm ae Roundup or 1830 ppm ae Kamba were used. aHerbicide+antibiotic mutation rates significantly different from LB but not from herbicide treatment, for all other combinations herbicide+antibiotic is significantly different from both LB and herbicide treatments. 'b. enterica experiments were conducted concurrently, using the same LB controls for both assays. E. coli was strain BW25113. ${ }^{\mathrm{CP}}<0.01{ }^{\mathrm{d} P}<0.001$ 
1

\begin{tabular}{lccc} 
& LB & LB+Herbicide & LB+Herbicide+Cip \\
\hline S. enterica & & & \\
Kamba & $3.57 \times 10^{-6}\left(1.27 \times 10^{-6}\right)^{\mathrm{b}}$ & $2.01 \times 10^{-4}\left(1.95 \times 10^{-4}\right)$ & $1.30 \times 10^{-2}\left(1.29 \times 10^{-2}\right)^{\mathrm{c}}$ \\
Roundup & $3.57 \times 10^{-6}\left(1.27 \times 10^{-6}\right)^{\mathrm{b}}$ & $2.91 \times 10^{-5}\left(2.47 \times 10^{-5}\right)$ & $2.79 \times 10^{-2}\left(1.71 \times 10^{-2}\right)^{\mathrm{ac}}$ \\
& & & \\
E. coli & & & \\
Roundup & $1.80 \times 10^{-9}\left(1.62 \times 10^{-9}\right)$ & $1.97 \times 10^{-10}\left(5.46 \times 10^{-11}\right)$ & $2.72 \times 10^{-5}\left(2.67 \times 10^{-5}\right)^{\mathrm{d}}$ \\
\hline
\end{tabular}

2 


\section{Table 3(on next page)}

Ciprofloxacin resistant variants of E. coli BW25113 per generation

$\mathrm{p}$-value for comparison between the treatment condition and LB medium. Kamba was used at $1830 \mathrm{ppm}$ ae, ciprofloxacin concentration was $0.025 \mu \mathrm{g} / \mathrm{mL}$ in liquid and $0.06 \mu \mathrm{g} / \mathrm{mL}$ in solid media. 
1

\begin{tabular}{lccc} 
Treatment & Resistant variants / generation & SEM & p-value \\
\hline LB & $7.5 \times 10^{-9}$ & $3.9 \times 10^{-9}$ & na \\
Kamba & $10^{-7}$ & $6.3 \times 10^{-8}$ & 0.22 \\
Cip & $1.2 \times 10^{-3}$ & $6.5 \times 10^{-4}$ & $2.4 \times 10^{-6}$ \\
Kamba+Cip & $5 \times 10^{-7}$ & $4.5 \times 10^{-7}$ & 0.27 \\
\hline
\end{tabular}

2 